\title{
High-resolution X-ray spectroscopy and imaging of supernova remnant N132D*
}

\author{
E. Behar ${ }^{1}$, A. P. Rasmussen ${ }^{1}$, R. G. Griffiths ${ }^{2}$, K. Dennerl ${ }^{3}$, M. Audard ${ }^{4}$, \\ B. Aschenbach ${ }^{3}$, and A. C. Brinkman ${ }^{5}$ \\ 1 Columbia Astrophysics Laboratory, 550 West 120th Street, New York, NY 10027, USA \\ 2 X-Ray Astronomy Group, Physics and Astronomy, University of Leicester, Leicester LE1 7RH, UK \\ 3 Max-Planck-Institut für Extraterrestrische Physik, 85740 Garching, Germany \\ 4 Laboratory for Astrophysics, Paul Scherrer Institute, Würenlingen and Villigen, 5232 Villigen PSI, Switzerland \\ 5 Space Research Organization of the Netherlands, Sorbonnelaan 2, 3548 CA, Utrecht, The Netherlands
}

Received 2 October 2000 / Accepted 30 October 2000

\begin{abstract}
The observation of the supernova remnant N132D by the scientific instruments on board the $X M M$ Newton satellite is presented. The X-rays from N132D are dispersed into a detailed line-rich spectrum using the Reflection Grating Spectrometers. Spectral lines of C, N, O, Ne, Mg, Si, S, and Fe are identified. Images of the remnant, in narrow wavelength bands, produced by the European Photon Imaging Cameras reveal a complex spatial structure of the ionic distribution. While K-shell Fe emission seems to originate near the centre, all of the other ions are observed along the shell. A high $\mathrm{O}^{6+} / \mathrm{O}^{7+}$ emission ratio is detected on the northeastern edge of the remnant. This can be a sign of hot ionising conditions, or it can reflect relatively cool gas. Spectral fitting of the CCD spectrum suggests high temperatures in this region, but a detailed analysis of the atomic processes involved in producing the $\mathrm{O}^{6+}$ spectral lines leads to the conclusion that the intensities of these lines alone cannot provide a conclusive distinction between the two scenarios.
\end{abstract}

Key words. atomic processes - line: formation - line: identification - techniques: spectroscopic - supernovae: individual: N132D - ISM: supernova remnants

\section{Introduction}

$\mathrm{N} 132 \mathrm{D}$, one of the brightest soft X-ray sources in the Large Magellanic Cloud (LMC), was identified as a type II supernova remnant (SNR) by Westerlund \& Mathewson (1966). The high velocities of the oxygen-rich (O-rich) ejecta near the centre of the remnant were first detected by Danziger \& Dennefeld (1976) and later measured precisely by Morse et al. (1995). The fast moving ejecta is believed to originate from a core collapse supernova (SN) of a massive star. Blair et al. (2000) have recently performed a thorough optical and UV study of N132D using the Hubble Space Telescope (HST). With the exceptionally high spatial resolution of the HST, they were able to distinguish between SN ejecta and swept-up interstellar matter (ISM). Observing a knot on the northwestern rim of the remnant, they found elemental abundances that agree fairly well with those previously obtained from

Send offprint requests to: E. Behar, e-mail: behar@astro.columbia.edu

* Based on observations obtained with XMM-Newton, an ESA science mission with instruments and contributions directly funded by ESA Member States and USA (NASA).
X-ray observations of the entire remnant (Hughes et al. 1998; Favata et al. 1998), and which are also consistent with the mean LMC abundances. In the ejecta, on the other hand, only $\mathrm{C}, \mathrm{O}, \mathrm{Ne}$, and $\mathrm{Mg}$ could be detected in the optical/UV spectra. The absence of O-burning elements, such as Si, S, and Fe, in the ejecta has led Blair et al. (2000) to suggest that N132D may be a product of a type Ib SN explosion.

N132D was first observed in the X-rays with the Einstein Observatory (Mathewson et al. 1983). Using the spectrometers on board Einstein, Hwang et al. (1993) detected individual emission lines of $\mathrm{O}^{7+}, \mathrm{O}^{6+}, \mathrm{Ne}^{9+}$, $\mathrm{Ne}^{8+}$, and $\mathrm{Fe}^{16+}$. Comparing with the ROSAT images, Morse et al. (1995) found little overlap between the optical and soft X-ray emitting regions. The X-ray emission seemed to come from a large shell of shocked ISM gas (see also Williams et al. 1999), which is also the source of strong synchrotron radio emission (Dickel \& Milne 1995). Hughes et al. (1998) fitted the ASCA CCD spectrum of N132D, obtaining mean LMC abundances, which further suggested that the X-rays in the remnant are predominantly emitted by shocked ISM. 
In this work, we present the X-ray spectrum of N132D measured with the Reflection Grating Spectrometers (RGS), complemented by the images taken with the European Photon Imaging Cameras (EPIC), both on board XMM-Newton. Owing to the uniquely high dispersion of the RGS, almost all of the spectral features emitted by this extended $\left(\sim 120^{\prime \prime}\right)$ source are resolved. Along with the RGS observation of 1E0102.2-7219 in the Small Magellanic Cloud (Rasmussen et al. 2001), these are the first highly resolved X-ray spectra of extended SNRs.

\section{Observation and data reduction}

The XMM-Newton X-ray observatory (Jansen et al. 2001) incorporates a payload with two identical high-resolution RGS spectrometers (den Herder et al. 2001) and three EPIC cameras (Turner et al. 2001; Strüder et al. 2001). The spectroscopic foci are separate from the telescope foci, where the cameras are located, allowing the cameras and the spectrometers to operate simultaneously. N132D was observed by XMM-Newton as part of the Performance Verification program during May, 2000. The RGS data were acquired with an effective exposure time of $53 \mathrm{ksec}$ for each spectrometer. The EPIC-MOS cameras were operated with the medium filter in place, giving effective exposure times of 18 and $21 \mathrm{ksec}$. The EPIC-PN camera was operated in full-frame mode using the medium filter for an effective exposure time of $23.2 \mathrm{ksec}$.

The RGS data were processed using custom software developed at Columbia University. The background subtracted CCD events were plotted in dispersion versus pulse-height space to separate the spectral orders. An especially large (cross-dispersion) spatial extraction of $135^{\prime \prime}$ was required in order to cover the extended remnant. In order to determine the flux and provide the channel-towavelength transformation, the count-rate spectra were folded through the response matrices specifically generated for this observation, utilizing the ground calibration (Rasmussen et al. 1998). This entire procedure was performed separately for each spectrometer and for first and second orders. Note that N132D's large angular size and velocity structure tend to broaden the spectral features irregularly, thus, preventing accurate wavelength measurements of individual lines. Fortunately, this does not impede the line identifications.

The reduction of raw EPIC-MOS event lists, which was performed using the standard Science Analysis Software, involves the subtraction of hot, dead, or flickering pixels, as well as removal of events due to electronic noise. EPIC-MOS images of the whole remnant were extracted using events with pattern 0-12 only. EPIC-PN data was reduced according to standard procedures. N132D covered the CCD chips 4 and 7, which required dedicated individual corrections. For the EPIC-PN images, areas of $44 \times 52$ pixels were extracted. The extraction area was adaptively increased around each pixel to contain at least 200 single-pixel events in the energy range of $4.4-10 \mathrm{keV}$.

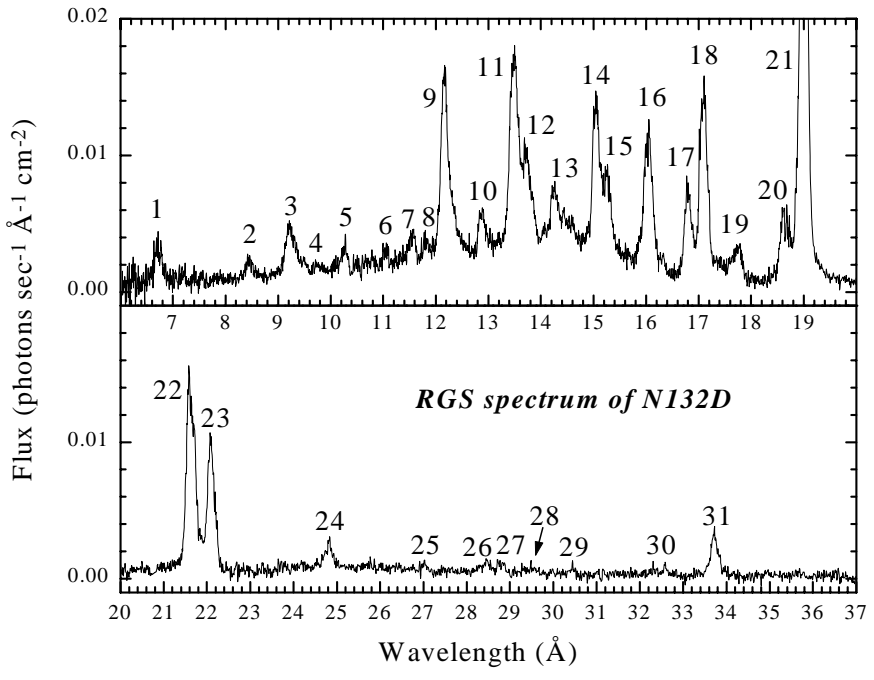

Fig. 1. The RGS fluxed spectrum of N132D. See Table 1 for line identifications

In order to get reliable statistics for the spectral fits, the data were rebinned to $0.16 \mathrm{keV}$ bins.

\section{Results}

\subsection{RGS spectrum}

The total RGS spectrum of N132D is plotted in Fig. 1. The data from the first and second orders in both RGS have been combined in order to improve the statistical reliability. The line identifications are presented in Table 1 for the strongest lines in accordance with the peak labels in Fig. 1. For blends, only the dominant contributing lines are given in the table. Lines of $\mathrm{C}, \mathrm{N}, \mathrm{O}, \mathrm{Ne}, \mathrm{Mg}, \mathrm{Si}, \mathrm{S}$, and $\mathrm{Fe}$ are unambiguously detected in the spectrum; the strongest lines originating from $\mathrm{K}$-shell $\mathrm{O}$ and $\mathrm{Ne}$, and Lshell $\mathrm{Fe}$. The ratio of the strong $\mathrm{Fe}^{16+}$ lines at $\sim 15$ and $17 \AA$ seem to indicate that the iron has equilibrated. At least six consecutive charge states of iron, namely $\mathrm{Fe}^{16+}$ $\mathrm{Fe}^{21+}$, are observed. $\mathrm{Fe}^{22+}$ might also be present in the $11.75 \AA$ blend (label 8). This wide range of Fe charge states indicates an electron temperature $\left(k T_{\mathrm{e}}\right)$ range of $0.2-1 \mathrm{keV}$ in collisional equilibrium (Mazzotta et al. 1998). The absence of $\mathrm{Fe}^{23+}$, which would have produced strong lines at $\sim 10.6$ and $11.2 \AA$, provides the upper limit for the temperature, whereas the lower limit is not well constrained as charge states lower than $\mathrm{Fe}^{16+}$ do not regularly emit $\mathrm{X}$-rays. However, line emission from $\mathrm{Fe}^{24+}$ is detected with EPIC-PN as discussed below.

Modeling the profiles and fluxes of the individual spectral lines in the RGS spectrum is a rather difficult task. The line profiles are correlated with the shape of the remnant, the velocity broadening, and the point spread function of the instrument (telescope + spectrometer). The fact that the former two effects may strongly depend on the ion species makes this problem especially challenging. Therefore, with the sole exception of the $\mathrm{O}^{6+}$ lines 
Table 1. Spectral lines in the RGS spectrum of N132D (Fig. 1). The references for the expected wavelengths $\left(\lambda_{\text {expected }}\right)$ are (1) Johnson \& Soff (1985), (2) Drake (1988), (3) Brown et al. (1998) (4) Brown et al. (2000) (5) Present HULLAC (Bar-Shalom et al. 1998) calculations

\begin{tabular}{|c|c|c|c|}
\hline Label & $\lambda_{\text {expected }}(\AA)$ & Reference & Ion \\
\hline \multirow[t]{3}{*}{1} & 6.648 & 2 & $\mathrm{Si}^{12+}$ \\
\hline & 6.685 & 2 & $\mathrm{Si}^{12+}$ \\
\hline & 6.739 & 2 & $\mathrm{Si}^{12+}$ \\
\hline 2 & 8.421 & 1 & $\mathrm{Mg}^{11+}$ \\
\hline \multirow[t]{3}{*}{3} & 9.169 & 2 & $\mathrm{Mg}^{10+}$ \\
\hline & 9.228 & 2 & $\mathrm{Mg}^{10+}$ \\
\hline & 9.314 & 2 & $\mathrm{Mg}^{10+}$ \\
\hline 4 & 9.708 & 5 & $\mathrm{Ne}^{9+}$ \\
\hline 5 & 10.238 & 5 & $\mathrm{Ne}^{9+}$ \\
\hline 6 & 11.009 & 5 & $\mathrm{Ne}^{8+}$ \\
\hline 7 & 11.554 & 5 & $\mathrm{Ne}^{8+}$ \\
\hline \multirow[t]{2}{*}{8} & 11.742 & 4 & $\mathrm{Fe}^{22+}$ \\
\hline & 11.770 & 4 & $\mathrm{Fe}^{21+}$ \\
\hline 9 & 12.135 & 1 & $\mathrm{Ne}^{9+}$ \\
\hline \multirow[t]{2}{*}{10} & 12.846 & 4 & $\mathrm{Fe}^{19+}$ \\
\hline & 12.864 & 4 & $\mathrm{Fe}^{19+}$ \\
\hline \multirow[t]{3}{*}{11} & 13.447 & 2 & $\mathrm{Ne}^{8+}$ \\
\hline & 13.518 & 4 & $\mathrm{Fe}^{18+}$ \\
\hline & 13.550 & 2 & $\mathrm{Ne}^{8+}$ \\
\hline 12 & 13.698 & 2 & $\mathrm{Ne}^{8+}$ \\
\hline 13 & 14.208 & 4 & $\mathrm{Fe}^{17+}$ \\
\hline 14 & 15.015 & 3 & $\mathrm{Fe}^{16+}$ \\
\hline 15 & 15.261 & 3 & $\mathrm{Fe}^{16+}$ \\
\hline \multirow[t]{2}{*}{16} & 16.005 & 1 & $\mathrm{O}^{7+}$ \\
\hline & 16.004 & 4 & $\mathrm{Fe}^{17+}$ \\
\hline 17 & 16.779 & 3 & $\mathrm{Fe}^{16+}$ \\
\hline \multirow[t]{2}{*}{18} & 17.051 & 3 & $\mathrm{Fe}^{16+}$ \\
\hline & 17.096 & 3 & $\mathrm{Fe}^{16+}$ \\
\hline 19 & 17.623 & 4 & $\mathrm{Fe}^{17+}$ \\
\hline 20 & 18.627 & 5 & $\mathrm{O}^{6+}$ \\
\hline 21 & 18.967 & 1 & $\mathrm{O}^{7+}$ \\
\hline \multirow[t]{2}{*}{22} & 21.602 & 2 & $\mathrm{O}^{6+}$ \\
\hline & 21.804 & 2 & $\mathrm{O}^{6+}$ \\
\hline 23 & 22.097 & 2 & $\mathrm{O}^{6+}$ \\
\hline 24 & 24.779 & 1 & $\mathrm{~N}^{6+}$ \\
\hline 25 & 26.988 & 5 & $\mathrm{C}^{5+}$ \\
\hline 26 & 28.464 & 5 & $\mathrm{C}^{5+}$ \\
\hline \multirow[t]{2}{*}{27} & 28.780 & 2 & $\mathrm{~N}^{5+}$ \\
\hline & 29.082 & 2 & $\mathrm{~N}^{5+}$ \\
\hline 28 & 29.535 & 2 & $\mathrm{~N}^{5+}$ \\
\hline 29 & 30.436 & 5 & $\mathrm{~S}^{13+}$ \\
\hline \multirow[t]{2}{*}{30} & 32.436 & 5 & $\mathrm{~S}^{13+}$ \\
\hline & 32.579 & 5 & $\mathrm{~S}^{13+}$ \\
\hline 31 & 33.734 & 1 & $\mathrm{C}^{5+}$ \\
\hline
\end{tabular}

discussed below, a detailed line-flux analysis is deferred to a later study.

\subsection{EPIC-MOS soft $X$-ray images}

Figure 2 shows the EPIC-MOS images of N132D in several narrow wavelength bands that overlap with those of the RGS. The data $\left(3^{\prime \prime} \times 3^{\prime \prime}\right.$ pixels $)$ in each band are combined from the two cameras, smoothed using a Gaussian function $\left(\right.$ sigma $\left.=1.5^{\prime \prime}\right)$, and then normalised to the brightest pixel in each image. Each narrow-band image is $2^{\prime} \times 2.5^{\prime}$ wide. All of the images feature strong X-rays from the shell, in particular from the southeastern, and northwestern edges. Differences between more and less ionised regions can be noticed. The emission by the most highly ionised species $\mathrm{Ne}^{9+}, \mathrm{Mg}^{10+}, \mathrm{Si}^{12+}$, and $\mathrm{Fe}^{19+}$ is brightest on the southeastern tip, while $\mathrm{O}^{6+}, \mathrm{O}^{7+}, \mathrm{Ne}^{8+}, \mathrm{Fe}^{16+}$, and $\mathrm{Fe}^{17+}$ exhibit strong emission on the northwestern rim as well. This is the ISM shocked region observed by Blair et al. (2000). $\mathrm{O}^{7+}$ emission is also intense at the centre of the remnant, indicating that part of the X-ray flux may be associated with the $\mathrm{SN}$ ejecta. The $\mathrm{O}^{6+}$ image is unique by virtue of the bright knot on the northeastern edge, just between the two broad inlets that extend into the ambient ISM. In fact, no other element seems to be emitting X-rays in that region. The EPIC-MOS ratio image of $\mathrm{O}^{6+} / \mathrm{O}^{7+}$ shown in Fig. 3 illustrates the excess of $\mathrm{O}^{6+}$ emission on the northeastern rim, where the ratio attains a value of $0.79 \pm 0.01$ in contrast with $0.60 \pm 0.01$ in the centre. The RGS dispersed images for these lines (not presented) are consistent with this picture. The $\mathrm{O}^{6+}$ brightness might simply be due to relatively cold gas. However, the special morphology in that region may, alternatively, suggest that the shock wave is interacting with relatively dense and cold O-rich material, possibly from pre-SN stellar winds. In that case, this newly shocked material can be very hot and ionising. It should be noted that Banas et al. (1997) have observed a giant molecular cloud near the southern edge of N132D, whereas no clouds were detected to the north of the remnant.

\subsection{EPIC-PN hard X-ray images}

The EPIC-PN camera is more sensitive to hard X-rays than the RGS and EPIC-MOS. The EPIC-PN CCD spectrum of the entire N132D remnant (not presented) shows $\mathrm{K}$-shell features of $\mathrm{Ar}$ and $\mathrm{Ca}$, as well as a prominent $\mathrm{Fe}^{24+}$ feature at $1.86 \AA(6.67 \pm 0.01 \mathrm{keV})$. This Fe-K feature was also observed with BeppoSAX (Favata et al. 1998). In Fig. 4a, flux images of N132D in this line (blend) are presented. In order to separate continuum from line flux, each spectrum was fitted with a Gaussian line superimposed on a bremsstrahlung continuum.

The N132D structure as seen in the EPIC-PN images (Fig. 4) is completely different from the structure in the EPIC-MOS images (Fig. 2). Unlike the softer emitting material that exhibits a shell with some filaments, the harder 


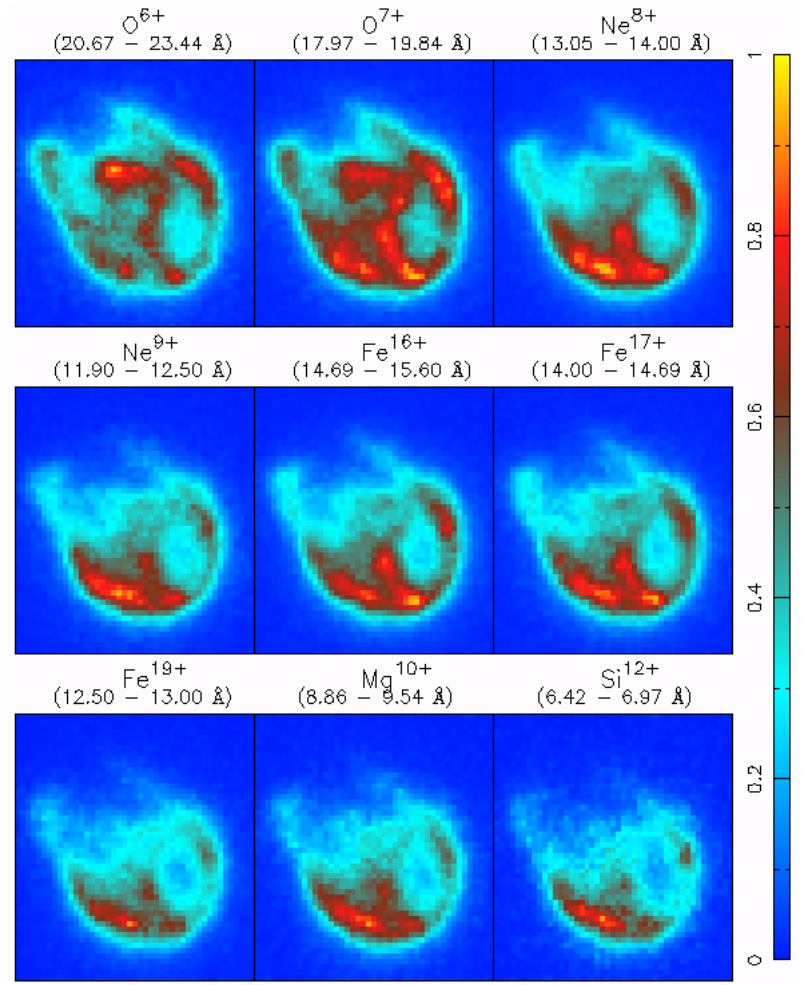

Fig. 2. EPIC-MOS images of N132D in the narrow wavelength bands indicated. Each image is labeled with the principal lineemitting ion in its particular band

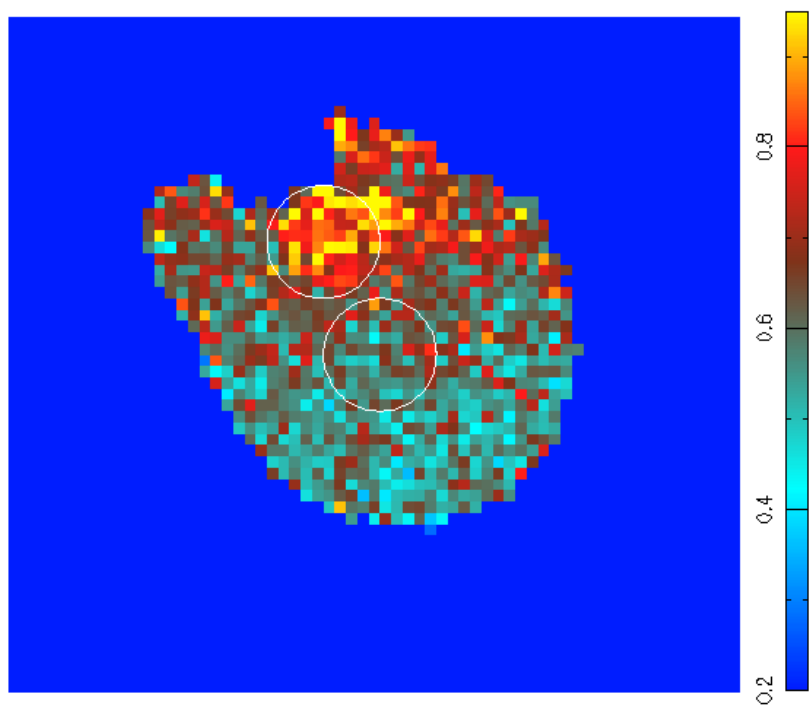

Fig. 3. EPIC-MOS $\mathrm{O}^{6+} / \mathrm{O}^{7+}$ ratio image of N132D. The average ratios in the circular regions $\left(15^{\prime \prime}\right.$ radius) are $0.79 \pm 0.01$ near the northeastern rim and $0.60 \pm 0.01$ in the centre

Fe-K line originates in a well-defined region slightly offset from the centre of the remnant to the southeast, where there is no sign for Fe-L emission. This is consistent with an out moving blast wave; the material closer to the centre that was shocked earlier is more highly ionised. If this material is associated with the SN ejecta, it might suggest that the high- $Z$ elements are not absent from the ejecta,
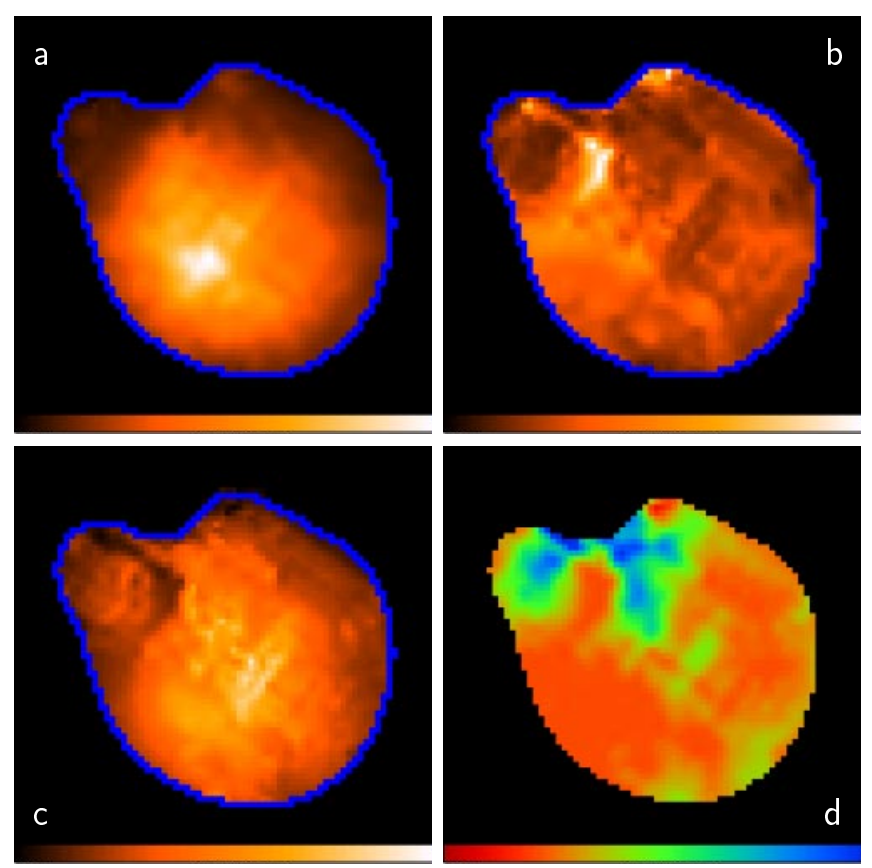

Fig. 4. N132D $150^{\prime \prime} \times 150^{\prime \prime}$ maps of a) the Fe-K line flux at $6.67 \mathrm{keV}$, (range 0-1.66 $10^{-5}$ photons $\mathrm{cm}^{-2} \mathrm{~s}^{-1} \operatorname{arcmin}^{-2}$ ), b) the EW of the Fe-K line, $(0-4.7 \mathrm{keV})$, c) the differential continuum flux at $6.67 \mathrm{keV}$ derived from the $4.4-10 \mathrm{keV}$ spectrum, (0-1.15 $10^{-5}$ photons $\left.\mathrm{cm}^{-2} \mathrm{~s}^{-1} \mathrm{keV}^{-1} \operatorname{arcmin}^{-2}\right)$, d) the apparent temperature $(0.4-3.1 \mathrm{keV})$ derived from the continuum only. All colour bars are linear

but rather that they could not be detected at shorter wavelengths because they are too ionised. The northwest and northeast regions show almost no $\mathrm{Fe}^{24+}$ emission. The continuum flux is highest near the centre (Fig. 4c), which makes N132D look like a centre-filled remnant, rather than a shell-type remnant, as suggested by the soft X-ray images. The highest equivalent width $(\mathrm{EW})$ values of more than a few $\mathrm{keV}$ are found in a confined northern region, where the Fe abundance is probably high and the overall density low. The hottest regions in N132D are found close to the northern inlets and are devoid of any Fe-K line emission, but do exhibit some continuum flux. This can be either due to depletion of iron in those regions, but can also be explained by the temperature exceeding $10 \mathrm{keV}$ or by a non-thermal continuum. Note that the bright $\mathrm{O}^{6+}$ spot (Fig. 2) seems to lie close to these hot regions, in which case $\mathrm{O}^{6+}$ would be definitely ionising.

In order to understand the absence of $\mathrm{Fe}^{23+}$ in the RGS spectrum and the weakness of $\mathrm{Fe}^{22+}$ and $\mathrm{Fe}^{21+}$, while emission from both lower and higher charge states is intense, the entire EPIC-PN spectrum above $2.5 \mathrm{keV}$ has been fitted with a two-temperature model. An acceptable fit is obtained for $k T_{\mathrm{e}}=0.89$ and $6.2 \mathrm{keV}$. These results can explain the absence of $\mathrm{Fe}^{23+}$, which attains its maximal fractional abundance at the intermediate temperature of $\sim 1.5 \mathrm{keV}$ (Mazzotta et al. 1998). The absence of these intermediate temperatures most likely means that the hot and cold regions are spatially distinct. 


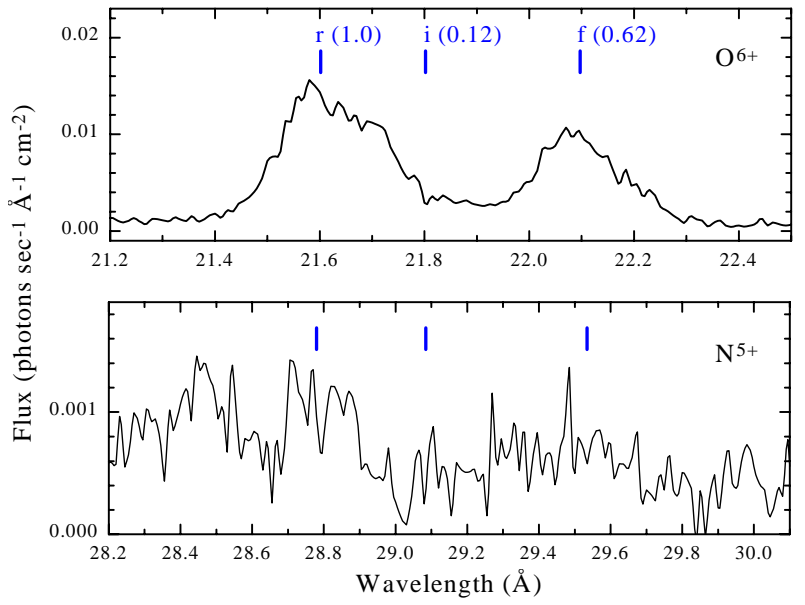

Fig. 5. The RGS spectrum of N132D in the limited wavelength range corresponding to the He-like triplets of $\mathrm{O}^{6+}$ and $\mathrm{N}^{5+}$ ( $\lambda_{\text {expected }}$ indicated by horizontal bars). Relative line intensities are given for $\mathrm{O}^{6+}$

\section{Spectroscopic analysis of the $\mathrm{O}^{6+}$ lines}

Seeking further evidence that the O-rich gas in the northeastern part of N132D is in the process of being ionised, we examine the RGS spectrum in the vicinity of the three strong emission lines of $\mathrm{O}^{6+}$ (He-like triplet). These lines are at $21.602,21.804$, and $22.097 \AA$ and are referred to, respectively, as the resonant $(r)$, intercombination $(i)$, and forbidden $(f)$ lines. The $i$ line is very weak in the N132D spectrum, whereas the $r$ and $f$ lines are strong, as can be seen in Fig. 5. A hint for similar line structure is found for the $\mathrm{N}^{5+}$ triplet as well (Fig. 5). Fitting the $\mathrm{O}^{6+}$ spectrum with the most appropriate line-profile model available yields line intensity ratios of $f: i: r=0.62: 0.12: 1.0$. However, since the model can not very well reproduce the observed line shapes, uncertainties of about $\pm 20 \%$ should be associated with these values. The quality of the data for $\mathrm{N}^{5+}$ is insufficient to allow for a similar measurement with the $\mathrm{N}^{5+}$ lines.

Decaux et al. (1997) pointed out that for He-like Fe, intense $r$ and $f$ lines together with a very weak $i$ line is an indication for hot ionising conditions, because: First, at high temperature the $r$ line is enhanced compared to the $i$ and $f$ lines. Second, inner-shell ionisation processes from the ground state $1 \mathrm{~s}^{2} 2 \mathrm{~s}$ of the Li-like ion to the $1 \mathrm{~s} 2 \mathrm{~s}(J=1)$ level enhance the $f$ line. In the present case of $\mathrm{O}$ and $\mathrm{N}$, inner-shell ionisation of Li-like ions also produces He-like excited ions in the $1 \mathrm{~s} 2 \mathrm{~s}$ configuration. This configuration has two levels; the $J=1$ level is the upper level of the $f$ line, whereas the $J=0$ level cannot decay radiatively to the ground level and in the case of Fe cannot decay radiatively at all. In contrast with the Fe case, for $\mathrm{O}$ and $\mathrm{N}$, the $1 \mathrm{~s} 2 \mathrm{p}_{1 / 2}(J=1)$ level, which is the upper level of the $i$ line, lies below the $1 \mathrm{~s} 2 \mathrm{~s}(J=0)$ level. This allows for radiative decay that enhances the $i$ line in parallel to the $f$-line enhancement.

We use atomic-state models employing data from the HULLAC code (Bar-Shalom et al. 1998) to calculate the spectrum of $\mathrm{O}^{6+}$ in a wide range of temperatures, both in ionising plasma and in equilibrium. Indeed, it is found that both situations can produce the observed $f: i: r$ ratios. Both hot (e.g., $0.6 \mathrm{keV}$ ) ionising gas with a rather low $\mathrm{O}^{6+} / \mathrm{O}^{5+}$ ratio (e.g., 2$)$, as well as a cooler $(0.15 \mathrm{keV})$ gas in equilibrium can emit these lines with the observed ratios. In other words, the intensities of the $\mathrm{O}^{6+}$ lines alone are insufficient for distinguishing between the equilibrium and ionising scenarios. For completeness, it should be noted that the current spectrum at least rules out a cooling plasma for N132D, because in that case, the upper levels of the $i$ and $f$ lines are populated by recombination resulting in much higher $f / r$ and $i / r$ ratios than are observed here.

\section{Conclusions}

The RGS spectrum of N132D features lines of C, N, O, $\mathrm{Ne}, \mathrm{Mg}, \mathrm{Si}, \mathrm{S}$, and Fe. K-shell Ar, Ca, and Fe are detected in the EPIC-PN spectrum. The narrow-band EPIC images of N132D give a coarse mapping of the elemental and temperature structure in the remnant. With the exception of $\mathrm{O}$, the dominant part of the soft X-ray emission originates from shocked ISM along the southeastern, and northwestern edges of the expanding shell. In contrast, strong Fe-K emission is detected near the centre of the remnant, perhaps indicating that high- $Z$ ejecta is too highly ionised to be observed in shorter wavelengths. $\mathrm{O}^{7+}$ is present throughout the remnant, while the brightest spot of $\mathrm{O}^{6+}$ emission is near the northeastern rim. The $\mathrm{O}^{6+}$ emission can be attributed, either to low temperatures, or to hot, recently shocked material that is in the process of being ionised. Fitting of the EPIC-PN spectrum indicates high temperatures in that region. Unfortunately, the emission lines of $\mathrm{O}^{6+}$ alone, although clearly seen in the RGS spectrum, cannot provide a conclusive distinction between these two scenarios, due to considerations associated with the atomic processes involved in producing these lines. A more detailed analysis of the RGS spectrum, and especially of the many strong Fe lines, will potentially provide a better insight into the temperature and non-equilibrium structure of the hot gas in N132D.

\section{References}

Banas, K. R., Hughes, J. P., Bronfman, L., \& Nyman, L.-Å. 1995, ApJ, 480, 607

Bar-Shalom, A., Klapisch, M., Goldstein, W. H., \& Oreg, J. 1998, The HULLAC code for atomic physics, unpublished

Blair, W. P., Morse, J. A., Raymond, J. C., et al. 2000, ApJ, 537,667

Brown, G. V., Beiersdorfer, P., Liedahl, D. A., Widmann, K., \& Kahn, S. M. 1998, ApJ, 502, 1015

Brown, G. V., Beiersdorfer, P., Liedahl, D. A., Widmann, K., \& Kahn, S. M. 2000, LLNL preprint [UCRL-JC-136647]

Danziger, I. J., \& Dennefeld, M. 1976, ApJ, 207, 394

Decaux, V., Beiersdorfer, P., Kahn, S. M., \& Jacobs, V. L. 1997, ApJ, 482, 1076

den Herder, J. W., Brinkman, A. C, Kahn, S. M., et al. 2001, A\&A, 365, L7 
Drake, G. W. 1988, Can. J. Phys., 66, 586

Dickel, J. R., \& Milne, D. K. 1995, AJ, 109, 200

Favata, F., Vink, J., Parmar, A. N., Kaastra, J., \& Mineo, T. 1998, A\&A, 340, 626

Hughes, J. P., Hayashi, I., \& Koyama, K. 1998, ApJ, 505, 732

Hwang, U., Hughes, J. P., Canizares, C. R., \& Market, T. H. 1993, ApJ, 414, 219

Jansen, F., Lumb, D., Altieri, B., et al. 2001, A\&A, 365, L1

Johnson, W. R., \& Soff, G. 1985, Atom. Data Nucl. Data Tables, 33, 405

Mathewson, D. S., Ford, V. L., Dopita, M. A., et al. 1983, ApJS, 51, 345

Mazzotta, P., Mazzitelli, G., Colafrancesco, S., \& Vittorio, N. 1998, A\&AS, 133, 403
Morse, J. A., Winkler, P., \& Kirshner, R. P. 1995, AJ, 109, 2104

Rasmussen, A., Cottam, J., Decker, T., et al. 1998, SPIE 3444, 327

Rasmussen, A. P., Behar, E., Kahn, S. M., van der Heyden, K., \& den Herder, J. W. 2001, A\&A, 365, L231

Strüder, L., Briel, U. G., Dennerl, K., et al. 2001, A\&A, 365, L18

Turner, M. J. L., Abbey, A., Arnaud, M., et al. 2001, A\&A, 365, L27

Westerlund, B. E., \& Mathewson, D. S. 1966, MNRAS, 131, 371

Williams, R. M., Chu, Y. H., Dickel, J. R., et al. 1999, ApJS, 123,467 